\title{
Factores sociolaborales asociados a la calidad de vida en el trabajo del profesional de enfermería que labora en una clínica privada, Lima, 2020
}

\author{
Socio-labor factors associated with the quality of life at work of the nursing \\ professional who works in a private clinic, Lima, 2020
}

Jaqueline Adanaqué Castillo', Aldeir Reynoso Valdez², Flor Lucila Contreras Castro³

\begin{abstract}
RESUMEN
Objetivo: determinar los factores sociolaborales asociados a la calidad de vida en el trabajo del profesional de enfermería que labora en una clínica privada de Lima. Metodologia: Es un estudio cuantitativo, correlacional, de corte transversal. La selección de la muestra se realizó mediante muestreo no probabilístico por conveniencia y estuvo conformada por 109 profesionales de enfermería. Para la recolección de datos sobre los factores sociolaborales, se utilizó un cuestionario elaborado por los investigadores y, para medir la calidad de vida, se utilizó el cuestionario "CVT-GOHISALO" propuesto por, Pando, González, Aranda y Elizalde (2017). Resultados: el 76,1\% de los profesionales de enfermería presenta Calidad de vida media en el trabajo, el 16,5\% alta, y el 7,3\% baja. En cuanto a la asociación de los factores sociolaborales y la calidad de vida en el trabajo se encuentra que el $39,4 \%$ con bajo apoyo social presenta calidad de vida baja, el $26,6 \%$ con alto apoyo social presenta calidad de vida alta; el $50,5 \%$ con alta discriminación, presenta calidad de vida baja, el 18,3\% que no sufre discriminación tiene calidad de vida alta; el $56 \%$ que percibe baja remuneración presenta calidad de vida baja, el $16,5 \%$ que percibe alta remuneración presenta calidad de vida alta; el $62,4 \%$ con deficientes condiciones de empleo presenta calidad de vida baja, el $20,2 \%$ con óptimas condiciones de empleo presenta calidad de vida alta; el $43,1 \%$ que percibe deficiente clima laboral presenta calidad de vida baja, y el $24,8 \%$ que percibe un óptimo clima laboral presenta calidad de vida alta. Los factores sociolaborales que presentan mayor asociación con la calidad de vida en el trabajo son: la discriminación y las condiciones de empleo con $\mathrm{OR}=4,672$ y OR=9,087 respectivamente. Conclusión: existe asociación significativa entre los factores sociolaborales y la calidad de vida en el trabajo de los profesionales de enfermería.
\end{abstract}

Palabras clave: Calidad de vida, factores sociolaborales, enfermería.

\section{ABSTRACT}

Objective: to determine the socio-labor factors associated with the quality of life at work of the nursing professional who works in a private clinic in Lima. Methodology: It is a quantitative, correlational, crosssectional study. The selection of the sample was made through non-probability sampling for convenience and was made up of 109 nursing professionals. To collect data on socio-labor factors, a questionnaire prepared by the researchers was used and, to measure quality of life, the questionnaire "CVT-GOHISALO" proposed by, Pando, González, Aranda and Elizalde (2017) was used. Results: $76.1 \%$ of the nursing professionals present Average quality of life at work, $16.5 \%$ high, and $7.3 \%$ low. Regarding the association of socio-labor factors and quality of life at work, it is found that $39.4 \%$ with low social support have low quality of life, $26.6 \%$ with high social support have high quality of life; $50.5 \%$ with high discrimination have a low quality of life, $18.3 \%$ who do not suffer discrimination have a high quality of life; $56 \%$ who receive low remuneration have low quality of life, $16.5 \%$ who receive high remuneration have high quality of

${ }^{1}$ Clínica Good Hope, Lima, Perú. Orcid ID: 0000-0003-1122-5179

${ }^{2}$ Clínica Good Hope, Lima, Perú.

Orcid ID: 0000-0002-4033-0595

${ }^{3}$ Universidad Peruana Unión, Lima,

Perú. Orcid ID: 0000-0002-4033-0595 
life; $62.4 \%$ with poor employment conditions present low quality of life, $20.2 \%$ with optimal employment conditions present high quality of life; $43.1 \%$ who perceive a poor work environment have a low quality of life, and $24.8 \%$ who perceive an optimal work environment have a high quality of life. The socio-labor factors that have the highest association with quality of life at work are: discrimination and employment conditions with OR $=4,672$ and $\mathrm{OR}=9,087$ respectively. Conclusion: there is a significant association between socio-labor factors and the quality of life in the work of nursing professionals.

Keywords: Quality of life, socio-labor factors, nursing.

\section{INTRODUCCIÓN}

La calidad de vida laboral se constituye en una percepción que expresan los trabajadores de una empresa sobre su grado de satisfacción o insatisfacción relacionado al medio ambiente en el que laboran, lo que evidencia el grado de bienestar y desarrollo que estas condiciones generan en los recursos humanos, la misma que cuando esta es negativa, impacta negativamente en el funcionamiento de la empresa (Cruz, 2018).

La calidad de vida profesional (CVP) es el sentimiento de bienestar que se deriva del equilibrio que el individuo percibe entre las demandas o cargas de la profesión y los recursos psicológicos, organizacionales y relacionales que dispone para afrontar estas demandas. Es un tema que viene siendo tratado en diversos ámbitos de estudios laborales especialmente para instituciones sanitarias, puesto que se han encontrado altos niveles de estrés en el personal de salud (Muñoz y Zenteno, 2017).

Con respecto a la profesión de enfermería, Baltodano y Mori (2014) mencionan que, en la actualidad, la enfermera asistencial está sometida a una sobrecarga laboral, debido a que los pacientes, cada vez, son más exigentes y de mayor complejidad. Sin embargo, por mucho tiempo se a evitado considerar puntos importantes relacionados a la calidad de vida de estos profesionales.

Es esencial considerar que una baja calidad de vida en el trabajo, puede ocasionar consecuencias negativas en la salud de los profesionales de enfermería, como el estrés, cansancio y agotamiento (Romero, Mesa y Galindo, 2010). Esto, se acrecienta debido a las exigencias sociales donde se necesita que los servicios hospitalarios estén en funcionamiento las 24 horas del día, con trabajo diurno y nocturno, para garantizar el cuidado del paciente en forma continua, lo que hace que los trabajadores, especialmente el profesional de enfermería, estén expuestos a condiciones duras de trabajo (Cabrera, Lluch, Peralta, y Granado, 2011).

Raeissi et al., (2019), realizaron un estudio en Irán "Calidad de vida laboral y factores asociados entre las enfermeras de los hospitales públicos" dicho estudio tuvo como objetivo determinar la calidad de vida laboral y los factores asociados de las enfermeras de los hospitales públicos de Irán. La metodología que se utilizó fue de estudio transversal en 2391 enfermeras de 85 hospitales públicos iraníes, seleccionado a través del muestreo por conveniencia. Los datos se recopilaron utilizando información demográfica y los cuestionarios sobre la calidad de vida laboral. Los resultados obtenidos fueron que la puntuación media de la calidad de vida laboral auto informada, con un $69.3 \%$ de las enfermeras insatisfechas con su vida laboral. Los principales factores que influyeron fueron los pagos inadecuados e injustos, la falta de solución de los problemas del personal por la organización y el apoyo deficiente de la administración, la inseguridad laboral, el alto estrés laboral, las políticas de promoción desleal y la participación inadecuada en la toma de decisiones. Se concluye que la calidad de vida laboral de enfermería fue de bajo nivel y necesita intervenciones de mejora.

Rivera y Lara, (2018) en México, "Calidad de vida laboral del personal de enfermería en una institución de salud privada", el objetivo fue evaluar el nivel de calidad de vida laboral del personal de enfermería en áreas de hospitalización de una institución de salud privada". Como de estudio cuantitativo, descriptivo transversal, pro lectivo, observacional. Realizada con 100 enfermeras (os) de hospitalización, se utilizó el Instrumento CTVGOHISALO con un Alpha de Cronbach de 0.89. Para el análisis estadístico inferencial se utilizó prueba t de student y Anova para comparación de más de dos grupos. Se tuvo como resultado que 
el $70 \%$ presentó un nivel medio de calidad de vida laboral, mientras que el 30\% manifiesta un nivel alto. Se concluyó que la evidencia demuestra que el personal de enfermería en las áreas de hospitalización en institución de salud privada en cuanto a su nivel de calidad de vida laboral se encuentra a partir del nivel medio.

Muñoz y Zenteno (2017) en Perú realizaron un estudio titulado, "Factores asociados a la calidad de vida profesional en enfermeros de un hospital público", el presenta estudio tiene como objetivo determinar los factores asociados a la calidad de vida profesional en enfermeros de un hospital. La metodología que se utilizó fue de tipo explicativo de corte transversal. La muestra estuvo conformada por 92 enfermeros. La variable dependiente fue la calidad de vida profesional. Se emplearon las pruebas de Chi-cuadrado y de $\mathrm{T}$ student para el análisis bivariado. Para medir la fuerza de asociación se utilizó la regresión logística de poisson calculando razón de prevalencias. Los resultados encontrados indican que el $51 \%$ tuvo una calidad alta. Así mismo los enfermeros que laboran en el servicio cirugía A tienen menos posibilidad de tener calidad alta (PR: 0.26 ). Se concluye que al laborar en el servicio cirugía " $A$ " está asociado con la CVP global baja. Asimismo, se encontró asociaciones significativas entre los factores laborales y las dimensiones de la CVP global.

Se realiza el presente trabajo de investigación con el objetivo de determinar los factores sociolaborales asociados a la calidad de vida en el trabajo del profesional de enfermería que labora en una clínica privada de Lima.

\section{METODOLOGÍA}

El estudio es de enfoque cuantitativo porque utiliza la recolección de datos para probar hipótesis de estudio, de diseño no experimental ya que no hubo manipulación de las variables. De tipo explicativo porque permite establecer relaciones causales obviando espurias, de corte transversal porque que se recolectaron datos en un solo momento (Hernández, et al., 2014).

\section{Participantes}

La población estuvo conformada por 226 profesionales de enfermería. La muestra estuvo conformada por 109 profesionales de enfermería; seleccionada mediante muestreo no probabilístico, por conveniencia, estableciéndose criterios de inclusión y exclusión.

\section{Instrumento}

Para medir la variable calidad de vida en el trabajo, se utilizó el instrumento denominado "CVT-GOHISALO" (versión breve) propuesto por, Pando, González, Aranda y Elizalde (2017). Consta de 31 ítems incluidos en siete categorías: soporte institucional en el trabajo; seguridad en el trabajo, integración al puesto de trabajo, satisfacción por el trabajo, bienestar logrado a través del trabajo, desarrollo personal del trabajador y administración del tiempo libre.

Las opciones de respuesta de cada ítem se encuentran en escala de Likert (nunca 0 , casi nunca 1 , algunas veces 2 , casi siempre 3 , siempre 4), con una puntuación de 0 a 4 . Las categorías consideradas para la calidad de vida en el trabajo: alta, media y baja.

Para la evaluación de la variable factores sociolaborales se utilizó el instrumento denominado "Factores sociolaborales" elaborado por los investigadores, teniendo como referencia las investigaciones de Lever, García, y Estrada (2013), en Colombia; Manzo y Moncallo (2004), en Venezuela; Squires, Fletcher, Hidalgo, y Nigenda (2018), en México y Crespo (2012), en España.

El cuestionario está conformado por 19 ítems, divididos en cinco dimensiones: apoyo social con 4 ítems, discriminación sociolaboral con 3 ítems; remuneración con 3 ítems, clima laboral con 5 ítems y condiciones de empleo con 4 ítems. Como opciones de respuesta se utiliza la escala de Likert (nunca 0 , casi nunca 1, algunas veces 2 , casi siempre 3 , siempre 4 ), que puntúan con una puntuación de 0 a 4 .

La confiabilidad del instrumento es de 0,849 según la prueba Alpha de Cronbach. Del mismo modo, se realizó la validación por juicio de 5 expertos, contando con un valor de $\mathrm{V}$ de Aiken de 0,9 .

Para el análisis univariado de las variables categóricas, se emplearon tablas de frecuencia, y para las variables numéricas se emplearon medidas de tendencia central y de dispersión.

Para el análisis bivariado, se elaboró tablas de 
contingencia y se aplicó la prueba estadística de chi-cuadrado.

Por último, para el análisis multivariado, se dicotomizó la variable calidad de vida en bueno (1) y malo (0), para realizar la regresión logística binaria, utilizándose la siguiente escala final de puntuación:

Malo (0): $30-75$

Buena (1): $76-120$

\section{Análisis de datos}

Los datos fueron procesados con el paquete estadístico IBM SPSS versión 26. Se procedió al ingreso y limpieza de la información considerando criterios de eliminación.

Al realizar la prueba de normalidad, a partir del estadístico Kolmogorv-Smirnov para muestras mayores de 50 , se obtuvo valor de $p$ inferiores a 0,05 , demostrando que no sigue una distribución normal.

Para el análisis univariado de las variables categóricas, se emplearon tablas de frecuencia, y para las variables numéricas se emplearon medidas de tendencia central y de dispersión.

Para el análisis bivariado, se elaboró tablas de contingencia y se aplicó la prueba estadística de chi-cuadrado.

Por último, para el análisis multivariado, se dicotomizó la variable calidad de vida en bueno (1) y malo (0), para realizar la regresión logística binaria, utilizándose la siguiente escala final de puntuación:

Malo (0): $30-75$

Buena (1): $76-120$

\section{RESULTADOS}

En la tabla 1 se observa que el $66,1 \%$ de la muestra presenta discriminación alta; el 68,8\% percibe remuneración baja; el 88,1\% manifiesta tener apoyo social alto; el $96,6 \%$ percibe un clima laboral óptimo y tener una condición de empleo óptimo.

Tabla 1

Factores sociolaborales de los profesionales de enfermería que laboran en una clínica privada, Lima, 2020

\begin{tabular}{lcc} 
Factores sociolaborales & $\mathrm{n}$ & $\%$ \\
Discriminación & 72 & 66,1 \\
Alta & 37 & 33,9 \\
Baja & & \\
Remuneración & 34 & 31,2 \\
Alta & 75 & 68,8 \\
Baja & & \\
Apoyo social & 96 & 88,1 \\
Alto & 13 & 11,9 \\
Bajo & & 96,6 \\
Clima laboral & 102 & 6,4 \\
Óptimo & 7 & \\
Deficiente & & 96,6 \\
Condiciones de empleo & 102 & 6,4 \\
Óptimo & 7 & \\
Deficiente & & \\
\hline
\end{tabular}

En la tabla 2 , se observa que el $76,1 \%$ de la muestra, presenta calidad de vida media en el trabajo, el $16,5 \%$ calidad de vida alta, y el $7,3 \%$ calidad de vida baja. 
Tabla2

Calidad de vida en el trabajo de los profesionales de enfermería que laboran en una cínica privada, Lima, 2020

\begin{tabular}{lll} 
Calidad de vida en el trabajo & $\mathrm{n}$ & $\%$ \\
Alta & 18 & 16,5 \\
Media & 83 & 76,1 \\
Baja & 8 & 7,3 \\
Total & 109 & 100 \\
\hline
\end{tabular}

En la tabla 3, a partir de la prueba chi cuadrado, se observa que 5 factores se relacionan a la calidad de vida en el trabajo del profesional de enfermería: apoyo social, discriminación sociolaboral, remuneración, clima laboral y condiciones de empleo, con un nivel de significancia de $p=0,000$.

El $39,4 \%$ de los profesionales de enfermería que manifiesta tener bajo apoyo social presenta calidad de vida baja en el trabajo, y el $26,6 \%$ con alto apoyo social presenta calidad de vida alta. El $50,5 \%$ de los profesionales de enfermería que manifiesta alta discriminación presenta calidad de vida baja en el trabajo y el 18,3\% que no sufre discriminación tiene calidad de vida alta.
El $56 \%$ de los profesionales de enfermería que refiere percibir baja remuneración, presenta calidad de vida baja en el trabajo y el $16,5 \%$ que indica tener una alta remuneración, presenta calidad de vida alta. El $62,4 \%$ de los profesionales de enfermería que indica tener deficientes condiciones de empleo presenta calidad de vida baja en el trabajo y el $20,2 \%$ que percibe óptimas condiciones de empleo presenta calidad de vida alta. Por último, el $43,1 \%$ de los profesionales de enfermería que la percibe un deficiente clima laboral, presenta calidad de vida baja en el trabajo; sin embargo, el $24,8 \%$ que percibe un óptimo clima laboral presenta calidad de vida alta.

Tabla3

Factores sociolaborales asociados con la calidad de vida en el trabajo de los profesionales de enfermería que laboran en una clínica privada, Lima, 2020

\begin{tabular}{|c|c|c|c|c|c|c|}
\hline \multirow[t]{3}{*}{ Factores sociolaborales } & \multicolumn{5}{|c|}{ Calidad de vida en el trabajo } & \\
\hline & \multicolumn{2}{|l|}{ Baja } & \multicolumn{2}{|l|}{ Alta } & \multirow[t]{2}{*}{ Sig. } & \\
\hline & n & $\%$ & n & $\%$ & & \\
\hline \multirow[t]{2}{*}{ Apoyo social } & Bajo & 43 & 39,4 & 3 & 2,8 & 0,000 \\
\hline & Alto & 34 & 31,2 & 29 & 26,6 & \\
\hline \multirow[t]{2}{*}{ Discriminación sociolaboral } & Alta & 55 & 50,5 & 12 & 11,0 & 0,001 \\
\hline & Baja & 22 & 20,2 & 20 & 18,3 & \\
\hline \multirow[t]{2}{*}{ Remuneración } & Baja & 61 & 56,0 & 14 & 12,8 & 0,000 \\
\hline & Alta & 16 & 14,7 & 18 & 16,5 & \\
\hline \multirow[t]{2}{*}{ Clima laboral } & Deficiente & 47 & 43,1 & 5 & 4,6 & 0,000 \\
\hline & Óptimo & 30 & 27,5 & 27 & 24,8 & \\
\hline \multirow[t]{2}{*}{ Condiciones de empleo } & Deficiente & 68 & 62,4 & 10 & 9,2 & 0,000 \\
\hline & Óptimo & 9 & 8,3 & 22 & 20,2 & \\
\hline
\end{tabular}

En la tabla 4, se observa que, los factores sociolaborales que tienen mayor asociación con la calidad de vida en el trabajo del profesional de enfermería son: la discriminación laboral y las condiciones de empleo. Los profesionales de enfermería que manifiestan discriminación sociolaboral alta, tienen 4 veces más probabilidades $(\mathrm{OR}=4,672)$ de presentar mala calidad de vida en el trabajo $(p=0,010)$ que aquellos que no perciben discriminación. Asimismo, el intervalo de confianza del Odds Ratio $(1,452$ - 15,034) no incluye a la unidad, lo que confirma su asociación.

De igual manera, los profesionales de enfermería que manifiestan deficientes condiciones de empleo tienen 9 veces más probabilidades $(\mathrm{OR}=9,087)$ de tener baja calidad de vida en el 
trabajo $(p=0,001)$ que aquellos que perciben óptimas condiciones de empleo. Así también, el intervalo de confianza del Odds Ratio $(2,452-33,671)$ no incluye a la unidad, lo que confirma la asociación.

Tabla 4

Análisis multivariado de los factores sociolaborales y la calidad de vida en el trabajo de los profesionales de enfermería que laboran en una clínica privada, Lima, 2020

\begin{tabular}{llllllllll} 
Variables en la ecuación & $B$ & E.T. & Wald & gl & Sig. Exp(B) & \multicolumn{2}{l}{ IC 95\% para EXP(B) } \\
\cline { 6 - 9 } & & & & & & & & Inferior & Superior \\
Apoyo social & 1,153 &, 829 & 1,937 & 1 &, 164 & 3,168 &, 624 & 16,068 \\
Discriminación laboral & 1,542 &, 596 & 6,682 & 1 &, 010 & 4,672 & 1,452 & 15,034 \\
Remuneración &, 400 &, 664 &, 362 & 1 &, 548 & 1,491 &, 405 & 5,485 \\
Clima laboral &, 562 &, 733 &, 586 & 1 &, 444 & 1,754 &, 417 & 7,382 \\
Condiciones de empleo & 2,207 &, 668 & 10,905 & 1 &, 001 & 9,087 & 2,452 & 33,671 \\
Constante & $-3,674$ &, 718 & 26,215 & 1 &, 000 &, 025 & & \\
\hline
\end{tabular}

${ }^{*} B=$ Parámetro estimado; $E . T .=$ Error estándar; Wald = Prueba estadística; gl = Grados de libertad; Sig. = Significancia (Margen de error); $\operatorname{Exp}(B)=$ Estimación de la OR (oods-ratio)

\section{DISCUSIÓN}

Aunque la práctica de enfermería puede ser significativa y satisfactoria personalmente, también es exigente y estresante, llegando a afectar el profesionalismo, desempeño y calidad de vida de los mismos, aumentando el riesgo de errores e incluso de una jubilación anticipada (Kumar, Bhat y Ryali, 2018).

En la presente investigación, se encontró que la mayoría de los profesionales de enfermería presenta calidad de vida media en el trabajo. Resultados similares, reportaron Rivera y Lara (2018), en Cuba; López (2018), en Perú; y Quintana (2014), en México, donde el 70\%, $84 \%$ y $41,7 \%$, respectivamente, presentaron una calidad de vida media laboral. Según Teles et al. (2014), cuando se percibe un desequilibrio entre el alto esfuerzo realizado en el trabajo y la baja recompensa de la misma, puede desarrollarse trastornos emocionales y consecuencias adversas para la salud, generando una mala calidad de vida en el trabajador. Si bien es cierto, en el presente estudio, la mayoría de los profesionales de enfermería presenta una calidad de vida media en el trabajo, existe el riesgo que descienda a mala calidad de vida ocasionando malos indicadores de atención hospitalaria, ausentismo laboral e insatisfacción del usuario (Seidl y Zannon, 2004). Por tanto, existe el reto de realizar esfuerzos para alcanzar una calidad de vida alta para aumentar el $16,5 \%$ obtenido en esta categoría.

También, se encontró relación significativa entre el apoyo social, discriminación sociolaboral, remuneración, clima laboral, condiciones de trabajo y la calidad de vida en el trabajo de los profesionales de enfermería.

Buenas condiciones de vida o circunstancias, determinarán la alta calidad de vida, pero si estas condiciones varían, la satisfacción del individuo con su calidad de vida también variará. La calidad de vida está influenciada por muchos factores y condiciones, tales como: alojamiento, empleo, ingresos, bienestar material, actitudes morales, vida personal y familiar, apoyo social, estrés y crisis, servicios de salud, condiciones de trabajo, alimentación, oportunidades educativas, relaciones con el medio ambiente, factores ecológicos y otros. Ruževičius, (2014)

Un estudio realizado por Grados (2017), evidencia que las condiciones de trabajo desfavorable condicionan una calidad de vida laboral insatisfecha en un $16.7 \%$, y que las condiciones laborales favorables condicionan una calidad de vida laboral satisfecha en un $34.4 \%$, concluyeron que existe relación significativa entre las condiciones de trabajo y la calidad de vida laboral de las enfermeras; así mismo, menciona que las enfermeras brindan beneficios a los seres humanos; sin embargo, por la falta de condiciones laborales adecuadas, los lleva a problemas actuales o alteraciones de conductas negativas o patológicas que conllevan en su estado de salud físico y mental, Canales, Valenzuela, y Paravic (2016). 
Según el análisis multivariado, las variables discriminación y condiciones de empleo tienen mayor asociación con la calidad de vida en el trabajo del profesional de enfermería con $p$ valor de 0,010 (OR: 4,672; IC: 1,452-5,034) y 0,001 (OR: 9,087; IC: 2,452-33,671), respectivamente. En torno a esto, Baptiste (2015) señala que las enfermeras ven la discriminación como un obstáculo para el avance y reconocimiento profesional, que afecta su bienestar físico y psicológico, la calidad de la atención al paciente y los costos de organización de la atención médica. Además, Wheeler, Foster y Hepburn (2014) afirman que, un ambiente de trabajo hostil interfiere con el desempeño laboral y afecta negativamente las oportunidades de empleo de un individuo. Asimismo, puede producir ansiedad, miedo, inseguridad, alienación, pérdida de autoestima y depresión. Para los empleadores, las consecuencias pueden ser malas actitudes en el trabajo, insatisfacción laboral, disminución de la moral, falta de confianza y aumento de la deserción, lo que debilita a la organización. Mejorar el empleo y las condiciones laborales atrae y retiene a los trabajadores de la salud, mientras también les permite desempeñarse de manera más efectiva, Oficina Internacional del Trabajo, (2017)

Los resultados demostrados contribuyen al desarrollo de múltiples estrategias por parte de los responsables de la institución y del personal de enfermería para mejorar la calidad de vida profesional. Las estrategias se perfilarán a tener mejoras en las condiciones de empleo de los profesionales considerando una buena distribución en los horarios rotativos de jornada laboral, pausas activas, etc. Por otro lado, mejorar en los factores de discriminación sociolaboral, tales como: asertividad y compañerismo entre los miembros del equipo multidisciplinario, trato justo entre supervisoras/coordinadoras de servicio, valoración por actos de buen servicio hacia los profesionales de enfermería. Todo esto tendrá una mirada positiva en la salud de los enfermeros, así como en la atención de calidad a los pacientes que son el motivo real del profesional de enfermería.

\section{Declaración de financiamiento y de conflictos de interés}

El estudio fue financiado por los autores, quienes declaran no tener algún tipo de conflicto de interés en la investigación realizada.

\section{Correspondencia}

Jaqueline Adanaqué Castillo

Correo electrónico:

jaquelineac@upeu.edu.pe

Aldeir Reynoso Valdez

Correo electrónico:

jesusreynosovaldez@gmail.com

\section{REFERENCIAS BIBLIOGRÁFICAS}

Baptiste, M. M. (2015). Workplace discrimination: An Additional Stressor for Internationally Educated Nurses. Online Journal of Issues in Nursing, 20(3), 1-15. Recuperado de: https://doi.org/10.3912/ OJIN.Vol20No03PPT01

Canales, M., Valenzuela, S., y Paravic, T. (2016). Condiciones de trabajo de los profesionales de enfermería en Chile. Enfermería Universitaria, 13(3), 178-186. Recuperado de:https://doi. org/10.1016/j.reu.2016.05.004

Crespo, J. L. (2012). Bienestar laboral en profesionales de la medicina. La doble cara de la organización sanitaria: Factores saludables y de riesgo psicosocial, 204.(Tesis para doctorado, Universidad Autonoma de Barcelona) Recuperado de: http://tdx.cbuc.es/handle/10803/284027

Cruz , E. (2018). La calidad de vida laboral y el estudio del recurso humano: una reflexión sobre su relación con las variables organizacionales. Revista científica Pensamiento y Gestión, 45. Recuperado de: https://doi.org/http://dx.doi. org/10.14482/pege.45.10617

Hernández Sampieri, R. (2014). Metodología de la investigación. MC Graw Hi (6ta edició). México: MC Graw Hi.

Kumar, A., Bhat, P., \& Ryali, S. (2018). Study of quality of life among health workers and psychosocial factors influencing it. Industrial Psychiatry Journal, 27(1), 96. https://doi.org/10.4103/ipj.ipj_41_18

Lever, J. P., García, G. L. M., \& Estrada, A. V. (2013). Elaboración de una escala de apoyo social (EAS) para adultos. Universitas Psychologica, 12(1), 129-137.

López, I. (2018). Calidad de vida y desempeño laboral del profesional de enfermería del servicio de centro quirúrgico del Hospital Cayetano Heredia, Lima-2018. (Tesis de maestría Universidad César Vallejo) Recuperado de: http://repositorio.ucv. 
edu.pe/bitstream/handle/20.500.12692/25867/ Bazualdo_FE.pdf?sequence $=1$ \&isAllowed $=y$

Manzo, E., y Moncallo, P. (2004). Remuneración salarial recibida, beneficios recibidos y aspirados relacionados con la calidad de vida laboral percibida. (Tesis de licenciatura, Universidad Católica Andrés Bello)

Muñoz, D. B., y Zenteno, P. (2017). Factores asociados a la calidad de vida profesional en enfermeros de un hospital público. Casus, 2(2), 89-99.

Oficina Internacional del Trabajo. (2017). Improving employment and working conditions in health services. Geneva.

Pando, M., González, R., Aranda, C., \& Elizalde, F. (2017). Fiabilidad y validez factorial del instrumento para medir calidad de vida en el trabajo "CVT-Gohisalo" (versión breve). Salud Uninorte, 34(1), 68-75. Recuperado de: https:// doi.org/http://dx.doi.org/10.14482/sun.34.1.9711

Phiangchai, P., Chureerat, S., \& Panu, O. (2017). Quality of Work Life Among Nurses. SciFed Nanotech Research Letters, 1(1), 200-206. Recuperado de: https://doi.org/10.23959/ sfnrl-1000005

Quintana, M. O. (2014). Calidad de vida en el trabajo, personal de enfermería. secretaría de salud pública, Hermosillo-México. UNIVERSIDAD DE CONCEPCIÓN.

Raeissi, P., Rajabi, M. R., Ahmadizadeh, E., Rajabkhah, K., \& Kakemam, E. (2019). Quality of work life and factors associated with it among nurses in public hospitals, Iran. Journal of the Egyptian Public, Health Association 94(1), 25. Recuperado de: https://doi.org/10.1186/s42506019-0029-2

Rivera Palacios, R., y Lara Barrón, A. (2018). Calidad de vida laboral del Personal de enfermería en una institución de salud privada. XVI Coloquio Panamericano de Investigación En Enfermería, 8.
Ruževičius, J. (2014). Quality of life and of Working Life: Conceptions and Research. Excellence in Services, 51(May), 317-334.

Seidl, E. M. F., \& Zannon, C. M. L. da C. (2004). Quality of life and health: conceptual and methodological issues. Cadernos de Saúde Pública / Ministério Da Saúde, Fundação Oswaldo Cruz, Escola Nacional de Saúde Pública. Recuperado de:https://doi. org/10.1590/S0102-311X2004000200027

Squires, A., Fletcher, J., Hidalgo, H. C., \& Nigenda, G. (2018). Un Estudio Piloto del Clima Laboral de las Enfermeras Mexicanas: A Pilot Study of Mexican Nurses' Work Environments. Hispanic Health Care International, 16(3), 145-157. Recuperado de: https://doi.org/10.1177/1540415318804481

Teles, M. A. B., Barbosa, M. R., Vargas, A. M. D., Gomes, V. E., e Ferreira, E. F., Martins, A. M. E. de B. L., \& Ferreira, R. C. (2014). Psychosocial work conditions and quality of life among primary health care employees: A cross sectional study. Health and Quality of Life Outcomes, 12(1), 72. Recuperado de: https://doi.org/10.1186/14777525-12-72

Recibido: 24/02/2019

Aceptado: 29/04/2020 